Special issue of the 3rd International Conference on Computational and Experimental Science and Engineering (ICCESEN 2016)

\title{
Numerical Computation of the Electrostatic Sheath Thickness
}

\author{
A. TAhraOui ${ }^{a, *}$, N. Fouial ${ }^{a}$ And B. ZAhAm ${ }^{a, b}$ \\ ${ }^{a}$ Quantum Electronics Laboratory, Faculty of Physics, \\ U.S.T.H.B., BP 32 El-Alia Bab-Ezzouar, Algiers 16111, Algeria
}

${ }^{2}$ Faculté des Sciences et des Sciences Appliquées, Université de Bouira, Rue Drissi Yahia 10000 Bouira, Algeria

\begin{abstract}
In this work we have established a one dimensional, stationary and non-magnetized theoretical model that describes the electrostatic sheath formation [M. Moisan, J. Pelletier, Physique des Plasmas Collisionnels, Application aux Décharges Haute Fréquence, EDP Sciences, 2006]. The sheath thickness is assessed. For this, we have assumed that all species are described by fluid equations. Dust grains are considered spherical particles with constant radii. Their charge is modeled by the orbit motion limited model [P.K. Shukla, A.A. Mamun, Introduction to Dusty Plasma Physics, Institute of Physics, Bristol 2001; A. Bouchoule, Dusty Plasmas, Physics, Chemistry and Technological Impacts in Plasma Processing, Wiley, Chichester 1999]. The solution of the obtained set of differential equations is found using the shooting method. The numerical results show that the sheath thickness depends considerably on the solid surface potential, as well as physical parameters, such as particle densities and temperatures, gas pressure, etc. The calculated electrostatic sheath thickness is greater than the thickness predicted by Child-Langmuir law [M.A. Lieberman, A.J. Lichtenberg, Principles of Plasma Discharges and Materials Processing, Wiley, New York 1994].
\end{abstract}

DOI: 10.12693/APhysPolA.132.1157

PACS/topics: 52.25.Vy, 52.27.Lw, 52.40.Kh

\section{Introduction}

The interaction of plasma with solid surfaces has received a great attention in the last three decades, as it is related to many physical phenomena, such as surface erosion, plasma processing, ion cyclotron heating, etc. This type of interaction is widely used in many areas of laboratory, industrial and astrophysical plasmas. Indeed, several researchers have investigated analytically and experimentally this subject [1-10]. This interaction gives rise to non-neutral region, called electrostatic sheath, in which a strong localized electric field occurs. Due to the higher mobility of electrons, against the ions mobility, the solid surface, such as electrode in discharge plasmas, acquires a negative potential with respect to the bulk plasma.

The presence of dust grains (in some cases, they are called impurities), ranging from nanometers to micrometers in size, leads to important modifications in the behavior of sheath and therefore remains an important and serious problem in plasma processing of integrated circuits, for example $[11,12]$. Thus, they change the plasma parameters and affect the collective processes, leading to the appearance of new modes and new mechanisms of damping and instabilities in such plasma systems.

For both laboratory and industrial plasmas, the electrostatic sheath thickness plays an important role in the bombardment or the erosion of the solid surface by accelerated particles present in the surrounding plasma. Furthermore, the electrostatic sheath thickness plays an important role in the trapping of impurities (dust grains)

*corresponding author; e-mail: alatif_tahraoui@yahoo.fr which constitute a brake of the fusion reaction in tokamaks, such as ITER (International Thermonuclear Experimental Reactor) for example.

Child and Langmuir [13] have computed the electrostatic sheath thickness in a particular case, where the plasma is considered collisionless and in absence of impurities. In recent works $[7-9,14]$, the electrostatic sheath is determined by the requirement that the electrons density is zero at the electrode (wall) position. In this work, we have proposed a new numerical approach, based on the shooting method, to compute numerically the electrostatic sheath thickness.

\section{Theoretical model}

We consider a $1 \mathrm{D}$, stationary and unmagnetized dusty low-pressure plasma model. The charged particles in the plasma electrostatic sheath are electrons $e$, ions $i$ and extremely heavy spherical dust grains $d$. The electrode, that is a plane solid surface with a negative electrostatic potential, is situated at position $z=L$. The sheath region lies between $z=0$ and the electrode, where $z$ is the position along the vertical axis, which is in the same direction as gravity $\boldsymbol{g}$. All particles $e, i$ and $d$ are described by fluid equations:

$$
\begin{aligned}
& \frac{\mathrm{d}\left(n_{e} v_{e}\right)}{\mathrm{d} z}=k_{i} n_{e} n_{n}, \\
& v_{e} \frac{\mathrm{d} v_{e}}{\mathrm{~d} z}=\frac{e}{m_{e}} \frac{\mathrm{d} \phi}{\mathrm{d} z}-\frac{1}{m_{e} n_{e}} \frac{\mathrm{d} p_{e}}{\mathrm{~d} z}-n_{n} \sigma_{e n} v_{e}^{2}, \\
& \frac{\mathrm{d}\left(n_{i} v_{i}\right)}{\mathrm{d} z}=k_{i} n_{e} n_{n}, \\
& v_{i} \frac{\mathrm{d} v_{i}}{\mathrm{~d} z}=-\frac{e}{m_{i}} \frac{\mathrm{d} \phi}{\mathrm{d} z}-\frac{1}{m_{i} n_{i}} \frac{\mathrm{d} p_{i}}{\mathrm{~d} z}-n_{n} \sigma_{i n} v_{i}^{2},
\end{aligned}
$$




$$
\begin{aligned}
& \frac{\mathrm{d}\left(n_{d} v_{d}\right)}{\mathrm{d} z}=0, \\
& m_{d} v_{d} \frac{\mathrm{d} v_{d}}{\mathrm{~d} z}=-q_{d} \frac{\mathrm{d} \phi}{\mathrm{d} z}+m_{d} g,
\end{aligned}
$$

where $\phi$ is the electrostatic potential, $m_{e, i}, n_{e, i}, v_{e, i}$, and $p_{e, i}$ are the mass, the density, the fluid velocity and the pressure of electrons and ions respectively, $n_{n}$ is the neutral gas density, $n_{d}, v_{d}, m_{d}$ and $q_{d}$ are the density, the fluid velocity, the mass and the charge of dust grains respectively, $k_{i}$ is the electronic impact ionization rate [13] and $\sigma_{e i, i n}$ are the electron-neutral and ion-neutral diffusion cross section [15], $e$ being the elementary charge.

The dust grain charge is due mainly to the electron and the ion fluxes, arriving to the dust grain,

$$
v_{d} \frac{\mathrm{d} q_{d}}{\mathrm{~d} z}=n_{i} e a_{i}-n_{e} e a_{e} .
$$

In the case of the orbit motion limited model [16-18], the attachment rates $a_{i}$ and $a_{e}$ are given by:

$a_{e}=\left\{\begin{array}{l}-\pi r_{d}^{2}\left(8 T_{e} / \pi m_{e}\right)^{1 / 2} \exp \left(e q_{d} / r_{d} T_{e}\right) \text { if } q_{d} \leq 0 \\ -\pi r_{d}^{2}\left(8 T_{e} / \pi m_{e}\right)^{1 / 2}\left(1+e q_{d} / r_{d} T_{e}\right) \text { if } q_{d}>0\end{array}\right.$

$a_{i}= \begin{cases}\pi r_{d}^{2} v_{i}\left(1-2 e q_{d} / r_{d} m_{i} v_{i}^{2}\right) & \text { if } q_{d} \leq 0, \\ \pi r_{d}^{2} v_{i} \exp \left(2 e q_{d} / r_{d} m_{i} v_{i}^{2}\right) & \text { if } q_{d}>0,\end{cases}$

where $r_{d}$ is the dust grain radius and $T_{e}$ is the electron temperature.

Finally, the electrostatic potential is determined by the Poisson's equation,

$$
\frac{\mathrm{d}^{2} \phi}{\mathrm{d} z^{2}}=-\frac{1}{\varepsilon_{0}}\left(n_{i} e-n_{e} e+n_{d} q_{d}\right),
$$

where $\varepsilon_{0}$ is the vacuum permittivity.

\section{Numerical results and discussion}

The main focus of this paper is the numerical computation of the electrostatic sheath thickness by using the shooting method. For this, argon is considered to be the background gas and the physical parameters used are: $T_{e}=2 \mathrm{eV}, T_{i}=0.05 \mathrm{eV}, n_{i 0}=10^{9} \mathrm{~cm}^{-3}, n_{n}=$ $10^{14} \mathrm{~cm}^{-3}, \sigma_{i n}=5 \times 10^{-15} \mathrm{~cm}^{2}, \sigma_{\text {en }}=5 \times 10^{-16} \mathrm{~cm}^{2}$, $u_{i 0}=v_{i 0} / c_{i s}=1.5, u_{d 0}=v_{d 0} / c_{d s}=2.5, r_{d}=1 \mu \mathrm{m}$, $\rho_{d}=2 \mathrm{~g} / \mathrm{cm}^{3}$ is the dust grains mass density, $c_{i s}=$ $\left(T_{e} / m_{i}\right)^{1 / 2}$ is the ion sound velocity, $c_{d s}=\left(z_{c} T_{e} / m_{d}\right)^{1 / 2}$ is the dust sound velocity and $z_{c}=r_{d} T_{e} / e^{2}$, where the subscript " 0 " denotes the equilibrium quantities $(\phi=0)$.

The set of Figs. 1a-d shows the effect of the solid surface (electrode) potential on the electrostatic sheath parameters and its thickness. Figure $1 \mathrm{~b}$ and $\mathrm{c}$ shows that the increase of the electrode potential makes the electron and ion density profiles stiffer and the sheath thickness becomes more important. Indeed, the increase of the solid surface potential increases the Coulomb repulsion, as well as the charge separation and consequently the sheath thickness.

In Fig. 1d, we have plotted the normalized sheath thickness $d / \lambda_{\mathrm{D}_{i}}$ versus the normalized solid surface potential $\Phi_{0}=-e \phi_{0} / T_{e}$, where $\lambda_{\mathrm{D}_{i}}$ is the ion Debye
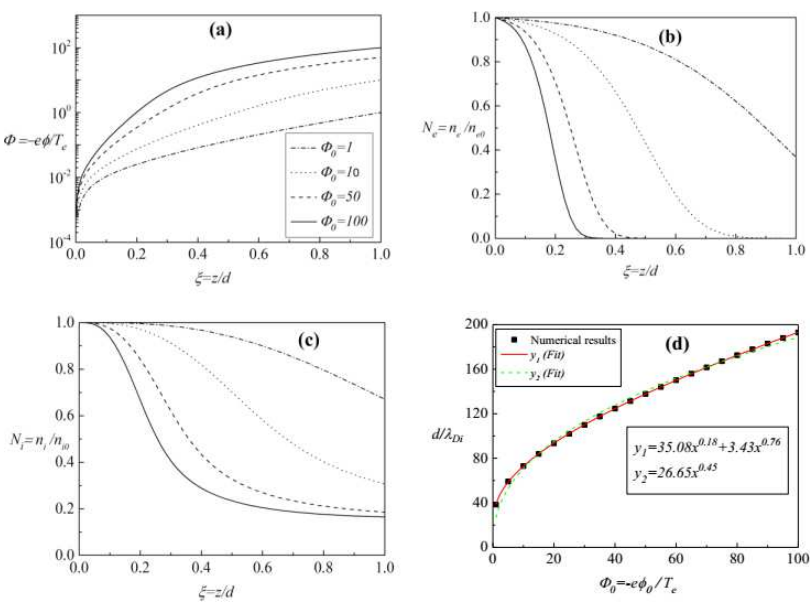

Fig. 1. Normalized electrostatic potential (a), normalized electrons density (b) and normalized ions density (c) versus the normalized spatial variable for different values of the normalized electrode potential. Normalized sheath thickness versus the normalized electrode potential $(d)$.

length. Unlike the Child-Langmuir law, which states that the electrostatic sheath thickness depends only on the electrode potential and the electrons temperature, i.e., $\left(d / \lambda_{D_{i}} \approx \Phi_{0}^{3 / 4}\right)$, the numerical results show that the electrostatic sheath thickness depends considerably on the solid surface potential and all other physical parameters, such as particle densities and temperature, gas pressure, etc. Furthermore, the computed electrostatic sheath thickness is significantly higher than that, derived by Child-Langmuir law. Finally, for practical uses, we have computed a numerical fit given by, $d / \lambda_{\mathrm{D}_{i}}=$ $35.08 \Phi_{0}^{0.18}+3.43 \Phi_{0}^{0.76}$.

\section{Conclusions}

The interaction of plasma with a solid surface (in our case, a plane electrode) and the formation of an electrostatic sheath is analysed analytically and numerically by using a fluid model. The electrostatic sheath properties, such as electrostatic potential and particle densities are computed and interpreted. In particular, the sheath thickness, that is a serious and important problem in the field of plasma-solid surface interaction, is computed exactly by using a shooting method. In contrast with the Child-Langmuir law, which indicates that the sheath thickness depends only on the electrode potential and electron temperature, it is found that in our case it depends strongly on all physical parameters and its value is higher than that of Child-Langmuir. For practical uses, the obtained results are summarized in a numerical fit.

\section{References}

[1] Jing Ou, Chunyun Gan, Binbin Lin, Jinhong Yang, Phys. Plasmas 22, 053705 (2015).

[2] M.M. Hatami, Phys. Plasmas 22, 043510 (2015). 
[3] Akihiro Kono, J. Phys. D: Appl. Phys. 37, 1945 (2004).

[4] R. Morales Crespo, R.N. Franklin, Plasma Sources Sci. Technol. 23, 035012 (2014).

[5] Wenqiu Li, Gang Wang, Dong Xiang, Xiaobao Su, Phys. Plasmas 24, 042103 (2017).

[6] S. Chekour, A. Tahraoui, B. Zaham, Phys. Plasmas 19, 053502 (2012).

[7] D. Benlemdjaldi, A. Tahraoui, R. Hugon, J. Bougdira, Phys. Plasmas 20, 043508 (2013).

[8] B. Zaham, A. Tahraoui, D. Benlemdjaldi, S. Chekour, Phys. Plasmas 21, 123702 (2014).

[9] D. Benlemdjaldi, A. Tahraoui, N. Fouial, S. Chekour, Acta. Phys. Pol. A 130, 11 (2016).

[10] N. Fouial, A. Tahraoui, R. Annou, Phys. Plasmas 23, 113702 (2016).

[11] L.I. Ryabova, D.R. Khokhlov, JETP Lett. 80, 133 (2004).
[12] S.G. Ovchinnikov, Zh. Eksp. Teor. Fiz. 102, 534 (1992); Sov. Phys. JETP 75, 283 (1992).

[13] M.A. Lieberman, A.J. Lichtenberg, Principles of Plasma Discharges and Materials Processing, Wiley, New York 1994.

[14] B.P. Pandey, A. Samarian, S.V. Vladimirov, Phys. Plasmas 14, 093703 (2007).

[15] B.M. Smirnov, Reference Data on Atomic Physics and Atomic Processes, Springer-Verlag, Berlin Heidelberg 2008.

[16] P.K. Shukla, A.A. Mamun, Introduction to Dusty Plasma Physics, Institute of Physics, Bristol 2001.

[17] A. Bouchoule, Dusty Plasmas, Physics, Chemistry and Technological Impacts in Plasma Processing, Wiley, Chichester 1999.

[18] A. Tahraoui, R. Annou, Phys. Plasmas 19, 014503 (2012). 